were compared using annualized incidence. The distribution of those flagged as HIV-positive was compared by database.

Results

The best performing case-definition (YI 0.71) was two or more HIV diagnoses in two years in physician claims, or in hospital discharge abstracts; or 14 or more HAART dispensations in two years; or one positive HIV laboratory. Sensitivity, specificity, PPV and NPV was 82.3\% (95\%CI: 79.1\%-85.5\%), 86.8\% (95\%CI: $84.9 \%-88.7 \%), \quad 74.1 \% \quad$ (95\%CI: $\quad 70.6 \%-77.6 \%)$, 91.4\% (95\%CI: 89.8\%-93.1\%), respectively. Annualized incidence (2009-2015) calculated from this case-definition was 7.4/100,000 persons (95\%CI: 6.8-8.1)]; annualized incidence calculated from surveillance data was 7.7/100,000 persons (95\%CI: 7.1-8.3). Approximately 76\% of cases would have been flagged through a positive laboratory; $43 \%$ through pharmaceutical claims; 34\% through physician claims; and 11\% through hospital abstracts. 95\% of cases would have been flagged through the combination of laboratory and pharmaceutical databases. Only $4 \%$ of cases were flagged in all four data sources.

Conclusion Although the combination of four databases produced the most complete prevalence snapshot, laboratory data was the most important contributor. The combination of laboratory and pharmaceutical databases would have identified the predominant majority of cases in our sample. Findings can be used to inform the construction of administrative data cohorts where the availability of population-based data sources may be more limited.

Disclosure No significant relationships.

\section{P207 BURDEN OF OPHTHALMIA NEONATORIUM AMONG BABIES OF PLHIV AT A DISTRICT HOSPITAL IN KUMASI, GHANA}

${ }^{1}$ Thomas Agyarko-Poku*, ${ }^{2}$ Alex Owusu Ofori, 3,4 Kwame Buabeng, ${ }^{5}$ Yaw Adu Sarkodie ${ }^{1}$ Suntreso Government Hospital, Ghana Health Service, Genitourinary Medicine, Kumasi, Ghana; ${ }^{2}$ Kwame Nkrumah University of Science and Technology, Kumasi Ghana; ${ }^{2}$ School of Medicine and Dentistry Sciences, College of Health Sciences, Kumasi, Ghana; ${ }^{3}$ Kwame Nkrumah University of Science and Technology, Kumasi Ghana; ${ }^{4}$ Department of Pharmacy Practice, Faculty of Pharmacy and Pharmaceutical Sciences, College of Health Sciences, Kumasi, Ghana; ${ }^{5}$ Kwame Nkrumah University of Science and Technology, Kumasi Ghana, School of Medicine and Dentistry Sciences, College of Health Sciences, Kumasi, Ghana

10.1136/sextrans-2019-sti.354

Background Ophthalmia neonatorium, also called neonatal conjunctivitis is a complication of Neisseria gonorrhoeae and Chlamydia trachomatis infections characterized by copious eyes discharge of newborn babies of infected untreated genital women. We examined babies born of women to determine the prevalence of ophthalmia noenatorium.

Methods This descriptive retrospective study reviewed the records of 257 babies aged 3 days to 14 days, of lactating women accessing care at the STI Clinic of the Suntreso Government Hospital in Kumasi Ghana from January to August 2018. Socio demographic characteristics as well as the clinical records and pregnancy details of the babies and mothers respectively were collected. Data was analysed using SPSS version 16.

Results $56(26.5 \%)$ of the 257 babies were from HIV positive mothers who have been on ART for over 2 years. $61.5 \%$ $(158 / 257)$ of the babies had uneventful delivery while $38.5 \%$
(99/257) were delivered through caesarean section on account of breach presentation and foetal distress. 47.3\% (122/257) of the mothers were symptomatic for vaginal discharge. A total of $211(82.1 \%)$ of the babies were diagnosed and received syndromic treatment for Ophthalmia neonatorium. Of this number $20.4 \%$ (43/211)) were babies of HIV positive mothers and represented $76.8 \%(43 / 56)$ of the total number of babies of the HIV positive mothers. The study found a significant association $(\mathrm{p},<0.000)$ between babies with Ophthalmia neonatorium (98/122) and symptomatic mothers as well as HIV infection $(\mathrm{p}<0.001)$.

Conclusion HIV infection is a risk factor for sexually transmitted infections Ophthalmia neonatorum remains a significant contributor to morbidity among babies born to Persons living with HIV. HIV positive women in the reproductive age group may have to be screened and treated for sexually transmitted infections in order to prevent further transmission to babies. Disclosure No significant relationships.

\section{P208 TESTING AND TREATING GENITAL AND EXTRA GENITAL BACTERIAL INFECTIONS IN HIV INFECTED PATIENTS: LESSONS LEARNED}

${ }^{1}$ Isabel Abreu, ${ }^{2}$ Luísa Graça, ${ }^{2}$ Joana Sobrinho Simões, ${ }^{2}$ Carmela Piñeiro, ${ }^{2}$ Jorge Soares, ${ }^{2}$ Rosário Serrão, ${ }^{2}$ Cátia Caldas*, ${ }^{2}$ António Sarmento. ${ }^{1}$ Centro Hospitalar São João, Infectious Diseases, Porto, Portugal; ${ }^{2}$ Centro Hospitalar e Universitário de São João, Infectious Diseases, Porto, Portugal

\subsection{6/sextrans-2019-sti.355}

Background Current evidence supports the screening of Neisseria gonorrhoeae (NG) and Chlamydia trachomatis (CT) in symptomatic disease in all sites with risk for carriage. The purpose is to increase the accuracy of diagnosis and prevent missed asymptomatic infections. This study aims to describe the incidence and aetiology of urethritis, proctitis and ulcers and compliance to additional screening.

Methods Retrospective study of three major syndromes diagnosed in a cohort of HIV positive patients (2430 patients) followed in a tertiary care hospital between July 2017 and June 2018.

Results We identified 86 patients with symptomatic infections. Most of them were in men who have sex with men. Twentynine $(34 \%)$ of these infections were urethritis. Almost half (48\%) were by NG, with $3(10 \%)$ additional NG infections detected in the anus and $6(21 \%)$ in the oropharynx. CT caused 7 cases (21\%) and in 2 cases it was detected exclusively in other sites. For all urethritis cases, 13 (48\%) weren't screened for NG/CT in the anus or oropharynx. Half of NG cases did not make a cure test. There were 17 proctitis: 6 (35\%) by NG and 9 (53\%) by LGV CT. Nine patients $(53 \%)$ didn't perform partner screening. Finally, there were 25 diagnosis of ulcers (23 genital; 2 oral). Eleven (44\%) had no identifiable cause and $8(32 \%)$ were primary syphilis. Of all cases, $11(44 \%)$ had no screening at other risk sites. More than half of the partners $(68 \%)$ were screened.

Conclusion The prevention of these infections through screening of all at risk sites, NG cure tests and partner screening can be challenging: it is time consuming, there may be limited interested in the treated patient for cure tests and the partners may be unknown or unwilling to be tested if asymptomatic. 